\title{
HEART
}

\section{Restoration of left ventricular synchronous contraction after acute myocardial infarction by stem cell therapy: new insights into the therapeutic implication of stem cell therapy for acute myocardial infarction}

S-A Chang, H-K Kim, H-Y Lee, S-Y Choi, B-K Koo, Y-J Kim, D-W Sohn, B-H Oh, Y-B Park, Y-S Choi, H-J Kang and H-S Kim

Heart 2008;94;995-1001; originally published online 1 Nov 2007; doi:10.1136/hrt.2007.124701

Updated information and services can be found at: http://heart.bmj.com/cgi/content/full/94/8/995

\section{References}

These include:

This article cites 25 articles, 16 of which can be accessed free at: http://heart.bmj.com/cgi/content/full/94/8/995\#BIBL

3 online articles that cite this article can be accessed at: http://heart.bmj.com/cgi/content/full/94/8/995\#otherarticles

\section{Rapid responses You can respond to this article at:} http://heart.bmj.com/cgi/eletter-submit/94/8/995

Email alerting Receive free email alerts when new articles cite this article - sign up in the box at the top right corner of the article

Topic collections Articles on similar topics can be found in the following collections

$$
\begin{aligned}
& \text { Drugs: cardiovascular system (9992 articles) } \\
& \text { Echocardiography (1470 articles) } \\
& \text { Acute coronary syndromes (1276 articles) } \\
& \text { Clinical diagnostic tests ( } 9110 \text { articles) }
\end{aligned}
$$

\section{Notes}




\title{
Restoration of left ventricular synchronous contraction after acute myocardial infarction by stem cell therapy: new insights into the therapeutic implication of stem cell therapy for acute myocardial infarction
}

\author{
S-A Chang, H-K Kim, H-Y Lee, S-Y Choi, B-K Koo, Y-J Kim, D-W Sohn, B-H Oh, \\ Y-B Park, Y-S Choi, H-J Kang, H-S Kim
}

\section{See Editorial, p 969}

Department of Internal Medicine, Cardiovascular Center, National Research, Laboratory for Cardiovascular Stem Cell, Innovative Research Institute for Cell Therapy, Seoul National University Hospital, Seoul, Korea

Correspondence to: Professor Hyo-Soo Kim, or Hyun-Jae Kang, Department of Internal Medicine, Seoul Natioal University Hospital, 28 Yongondong, Chongno-gu, Seoul, 110744, Korea; hyosoo@snu.ac.kr or nowkang@snu.ac.kr

Accepted 4 September 2007 Published Online First

1 November 2007

\begin{abstract}
Objective: To evaluate the effects of stem cell therapy on restoration of the left ventricular (LV) synchronous contraction in patients with acute myocardial infarction (AMI).

Methods: 40 patients with AMI who underwent successful coronary revascularisation were randomly allocated to the cell infusion or the control group. Evaluations were performed with echocardiographic tissue synchronisation imaging to determine LV dyssynchrony and with cardiac magnetic resonance imaging to estimate LV ejection fraction (LVEF) at baseline and at 6 months. To quantify the severity of systolic LV dyssynchrony, the standard deviations of time to peak systolic velocity of the $12 \mathrm{LV}$ segments (TS-SD) were calculated.
\end{abstract}

Results: At 6 months, greater improvements of Ts-SD ( $\Delta$ Ts-SD: -45.0 (40.2) vs 5.0 (39.9) ms, $p<0.001)$ and LVEF ( $\Delta$ LVEF: $6.8 \%(9.1 \%)$ vs $-0.2 \%(6.9 \%), p=0.015)$ relative to the corresponding baseline values were observed in the cell infusion group than in the control group. By multivariate analysis, $\Delta T S-S D$ and baseline LVEF emerged as the independent determinants of LVEF improvement and cell infusion, and baseline Ts-SD as the determinant of $\Delta \mathrm{Ts}$-SD improvement. Maximal exercise capacity measured by symptom-limited treadmill testing correlated well with Ts-SD but not with LVEF at 6 months of follow-up.

Conclusion: Stem cell therapy had a favourable effect on the restoration of LV synchronous contraction in patients with AMI.

An accumulated body of evidence suggests that synchronous contraction of the heart is one of the most important factors in maintaining cardiac output or stroke volume in concert with adequate heart rate, preload and afterload. ${ }^{1}$ Cardiac resynchronisation therapy has been recently developed and has been shown to guide simultaneous left ventricular (LV) contraction and consequently to improve haemodynamic status, exercise performance and prognosis in selected patients. ${ }^{2}$

Recently, the beneficial effect of peripheral blood-derived stem cell treatment on recovery of the LV systolic performance in myocardial infarction has been well demonstrated. ${ }^{4-7}$ Despite the favourable effect of stem cell therapy on LV systolic function, no data are available regarding the role of stem cell therapy in improving LV synchronous contraction in patients with acute myocardial infarction (AMI).

Therefore, we sought to evaluate, in this prospective clinical study using novel echocardiographic tissue synchronisation imaging technique, ${ }^{8}$ (1) whether stem cell therapy can lead to a recoordination of LV systolic contraction and, if so, (2) whether LV systolic function and exercise capacity might benefit from improved LV harmonious contraction.

\section{METHODS}

\section{Patient population}

To establish a normal range for the index of LV synchronicity, we consecutively recruited 129 healthy normal volunteers (86 males, 46 (SD 12; range 19-69) years). The volunteers had no previous medical history and had normal physical and laboratory examinations including electrocardiography, echocardiography and either treadmill test or coronary computed tomographic angiography.

We have previously reported a 6-month followup. ${ }^{6}$ We started the tissue synchronisation imaging study in the middle of enrolment for MAGIC Cell3-DES trial and continued after completion of enrolment for this trial. In addition to $18 \mathrm{AMI}$ patients from the MAGIC Cell-3-DES trial, 22 patients who were randomised and treated under the same protocol were enrolled for this tissue synchronisation imaging study. Thus, a total of 40 AMI patients were randomised into either the cell infusion or the control group.

Patients with successful drug-eluting stent (DES) implantation in the culprit lesion after AMI were eligible for this study. In addition to the exclusion criteria of the MAGIC Cell-3-DES trial, ${ }^{6}$ patients with atrial fibrillation or with an implanted pacemaker were also excluded in the current study.

\section{Study protocol}

The study was performed using the MAGIC Cell-3DES trial protocol and details have been previously described. ${ }^{6}$ Briefly, patients were randomly allocated to either the cell infusion or the control group. Cardiac magnetic resonance imaging (MRI), transthoracic echocardiography and treadmill 


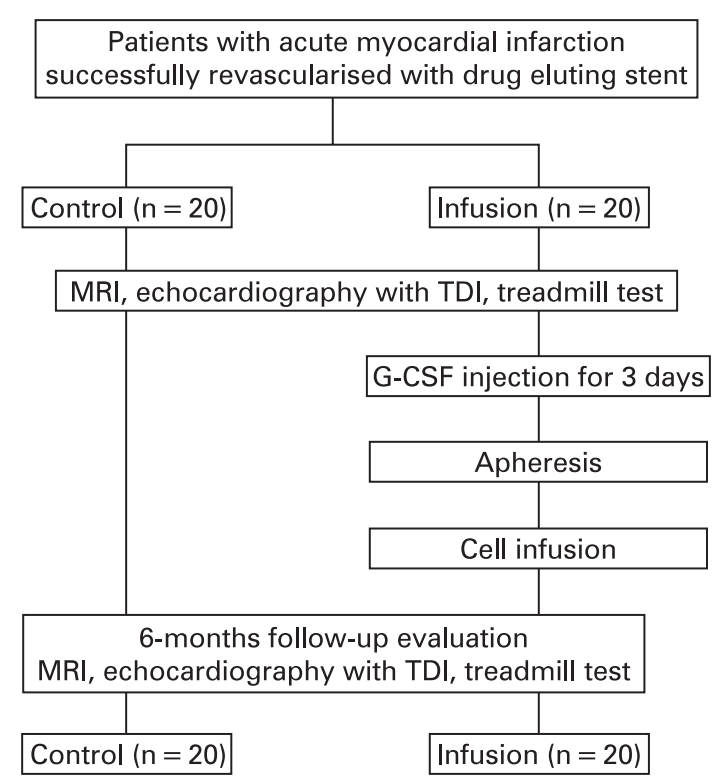

Figure 1 Study protocol. MRI, magnetic resonance imaging; TDI, tissue Doppler imaging; G-CSF, granulocyte-colony-stimulating factor.

testing were performed at baseline after DES implantation and at the 6-month follow-up. For patients assigned to the cell infusion group, granulocyte-colony-stimulating factor (G-CSF, Dong-A Pharmaceutical, Seoul, Korea) was administered daily subcutaneously for a consecutive 3 days at $10 \mu \mathrm{g} / \mathrm{kg}$ body weight to mobilise stem cells, and then the mobilised stem cells were collected using a Cobe spectra apheresis system (Cobe BCT Inc, Lakewood, CO, USA) using the mononuclear cell collection method. The infusion doses were $1 \sim 2 \times 10^{9}$ monocytes per patient, guaranteeing a minimum target cell dose of $7 \times 10^{6} \mathrm{CD} 34+$ cells. Stem cells collected were selectively infused into the coronary artery supplying infarcted myocardium via an over-the-wire coronary balloon catheter (fig 1).

The study protocol was approved by the institutional review board of Seoul National University Hospital. Written informed consent was obtained from all patients after an in-depth explanation of the procedure and the study risks.

\section{Echocardiographic data acquisition and analysis}

Transthoracic echocardiography was conducted using a commercially available device (Vivid 7, GE Medical System, Horten, Norway) at baseline and 6-month follow-up. After standard echocardiographic examination, Doppler tissue imaging (DTI) mode was activated in apical four-chamber, two-chamber and long-axis views. Great care was taken to get the highest possible frame rate with sector width and depth optimised. Three consecutive heart beats were digitally saved in a cineloop format by two independent echocardiographers for later offline analysis with a dedicated software package (EchoPac 5.0.1, GE Medical System). Offline analyses were performed by another echocardiographer, who is not involved in image acquisition or patients' clinical follow-up and who did not know patients' clinical data.

This tissue synchronisation imaging (TSI) algorithm automatically detects peak positive systolic velocity within a prespecified time interval, and displays activation differences in time to peak positive systolic velocity (Ts) between myocardial segments through colour-coding directly superimposed on twodimensional images, which ranges from green (earliest arrival at peak systolic velocity), yellow, orange to red (latest arrival at peak systolic velocity). ${ }^{8}$ For the measurement of Ts values, the onset of a ORS signal on the electrocardiogram was used as a reference point. In TSI mode, we could easily obtain the Ts values of myocardial segments by simply placing a $6-\mathrm{mm}$ diameter region of interest in the middle of the segment concerned. To estimate LV systolic dyssynchrony, we employed the six-basal and six-mid-segmental model in the LV (total 12 LV segments). After the procurement of the Ts values of $12 \mathrm{LV}$ myocardial segments, Ts standard deviation (Ts-SD) was automatically calculated. We adopted Ts-SD of the $12 \mathrm{LV}$ segments as a surrogate index for LV synchronicity because TsSD obtained using either conventional DTI or TSI tools was found to be the most reliable index of LV synchronicity ${ }^{90}$ that is, the greater the value of $\mathrm{Ts}-\mathrm{SD}$, the more severe the systolic dyssynchrony. The average Ts-SD values from three consecutive heart beats were taken for analysis. In addition, the global peak systolic myocardial velocity of LV ( $\left.S^{\prime}\right)$ was calculated from mean peak systolic velocities from six-basal segments as a representative marker for the LV inotropic state. $\Delta \mathrm{Ts}-\mathrm{SD}$ was defined as the Ts-SD at 6-month follow-up minus Ts-SD at baseline.

Inter-observer and intra-observer variabilities for Ts-SD measurements were determined by analysing 10 randomly selected patients by two independent blinded observers. Data were analysed by least-squares-fit linear regression analysis using standard-error-of-estimate (SEE).

\section{Cardiac magnetic resonance imaging}

Cardiac MRI (Sonata 1.5T, Siemens, Erlangen, Germany) was performed at baseline and at 6 months. Short axis cine images with a slice thickness of $8 \mathrm{~mm}$ and a gap of $2 \mathrm{~mm}$, were acquired throughout the entire LV using contiguous twodimensional steady state precession sequences. Using ARGUS software (Siemens, Erlangen, Germany), LV volumes and LV ejection fraction (LVEF) were calculated by a blinded observer.
Figure 2 Comparisons of changes in left ventricular ejection fraction ( $\triangle \mathrm{LVEF}$ ) or Ts-SD changes ( $\triangle \mathrm{Ts}$-SD) between the control and cell infusion groups. $(A)$ In the cell infusion group, LVEF improvements were significantly greater than in the control group. (B) The cell infusion group showed a remarkable reduction in $\Delta$ TsSD compared to the control group, indicating a better improvement in left ventricular synchronous contraction in the cell infusion group.
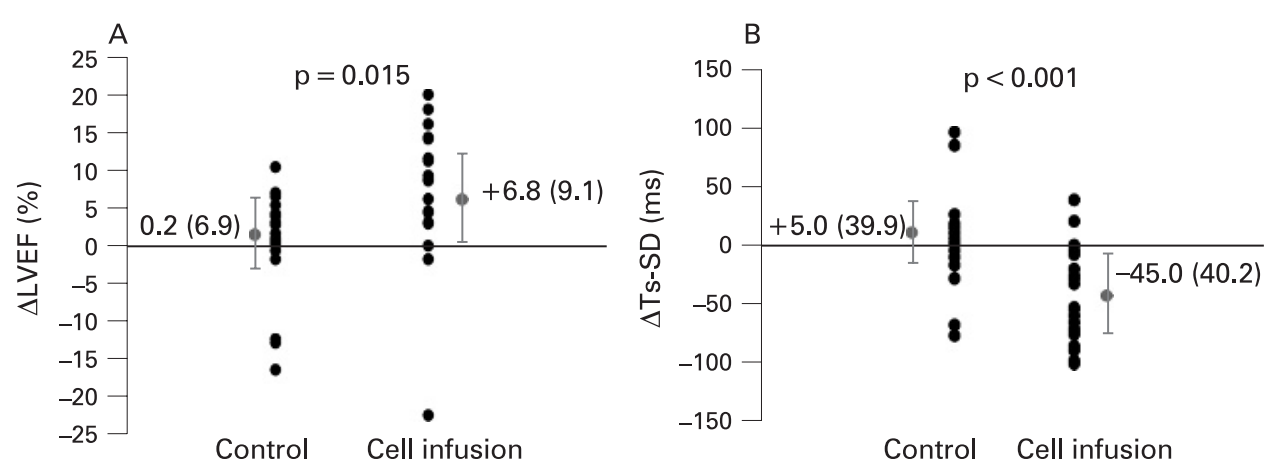
$\triangle$ LVEF was defined as LVEF at 6-month follow-up minus LVEF at baseline.

\section{Treadmill exercise test}

Patients underwent a maximum symptom-limited treadmill testing using the modified Bruce protocol at 1 week and 6 months after revascularisation. All anti-anginal medication was continued. The end points of this test included angina pectoris, fatigue, dyspnoea and a significant decrease in blood pressure of more than $10 \mathrm{~mm} \mathrm{Hg}$. The achievement of a maximal age-related heart rate was not a reason for termination. Exercise capacity was measured using metabolic equivalents (METs), an estimate based on treadmill speed and grade.

\section{Statistical analysis}

All values are expressed as means (SD) or as percentages. To compare parametric variables at baseline and at 6-month follow-up, we used the paired t test or Wilcoxon signed rank sum test as appropriate. Comparisons of the continuous variables between the cell infusion and control groups were performed using the independent t test, whereas Fisher's exact test was used for categorical variables. Pearson's correlation analysis was employed to compare the relation between $\Delta T$ sSD and $\triangle$ LVEF after the 6-month follow-up and the relation between Ts-SD and exercise duration at the 6-month follow-up. Multivariate backward stepwise logistic regression analyses were undertaken to identify independent determinants of $\triangle$ LVEF and $\triangle T$ S-SD. All statistical analyses were performed with SPSS 12.0 (SPSS Inc, Chicago, IL, USA), and $p$ values of $<0.05$ were taken to indicate statistical significance.

\section{RESULTS \\ Study population}

Mean Ts-SD obtained from 129 normal subjects was 23.9 (11.2) $\mathrm{ms}$ and, hence, a Ts-SD of $46 \mathrm{~ms}$ (mean (+2 SD) for normal subjects) was chosen as a cut-off upper limit value to define LV synchronous contraction. Since no significant change in Ts-SD was detected with ageing $(p=0.75)$, no further correction for age was carried out in the following analyses.

All the patients underwent the apheresis process without immediate complication and were successfully given stem cell infusion, which contains $9.04(8.04) \times 10^{7} \mathrm{CD} 34+$ cells and

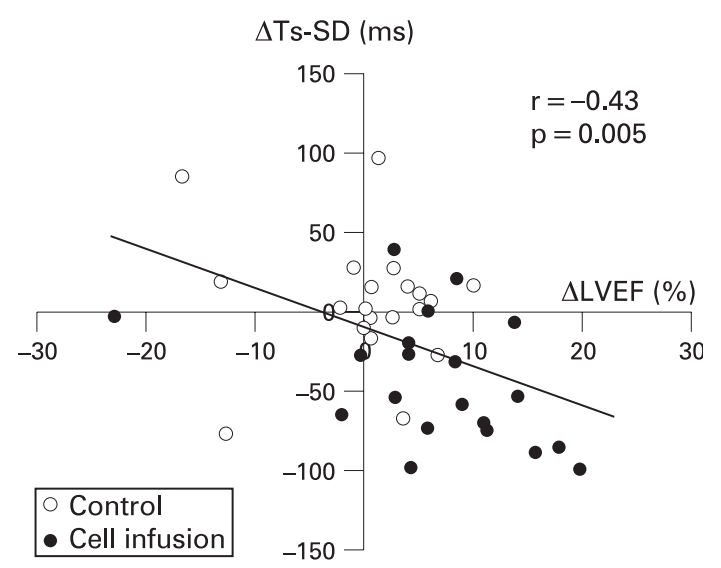

Figure 3 Scatter plots for correlations between changes in left ventricular ejection fraction ( $\triangle \mathrm{LVEF}$ ) and Ts-SD changes ( $\triangle \mathrm{T} s-\mathrm{SD}$ ) after 6-month follow-up in all patients, in the control and in the cell infusion groups. Significant negative correlations were noted for all patients and for the cell infusion and control group, respectively. completed the follow-up at 6 months. Among them, 35 performed the exercise test, the other five complained of leg pain caused by degenerative arthritis or claudication. Baseline clinical, echocardiographic and cardiac MRI data of the patient cohort are summarised in table 1.

There was a wide range of variation in Ts-SD and LVEF at baseline in both the control and the cell infusion groups, but there were no significant differences between the two groups in terms of baseline characteristics. When a Ts-SD of $>46 \mathrm{~ms}$ was used to define significant systolic asynchrony, it was found to be present in 15 patients (75\%) in the control group and in 17 patients $(85 \%)$ in the cell infusion group $(p=0.70)$, indicative of the presence of LV dyssynchrony in a large proportion of the patients. Again, baseline characteristics were similar between patients with (responders) and those without (non-responders) an improvement in LV dyssynchrony except for the presence of a higher proportion of patients with cell infusion treatment in the responders group (table 1 ).

\section{Improvement of Ts-SD and LV systolic function by stem cell therapy}

At the 6-month follow-up, LVEF increment from baseline was significantly higher in the cell infusion group, compared to the control group (ALVEF: $6.8 \%$ (9.1\%) vs $0.2 \%(6.9 \%), p=0.015)$ (fig 2). Ts-SD values were markedly improved at the 6-month follow-up than at baseline in the cell infusion group (108.0 (50.1) ms vs 63.0 (54.9) ms, $\mathrm{p}<0.001)$, but not in the control group (98.2 (52.8) ms vs 103.2 (50.1) ms, p=0.59) (table 2), implying that $\Delta \mathrm{Ts}$-SD improvement was greater in the cell infusion group than in the control group (-45.0 (40.2) ms vs 5.0 (39.9) ms, $\mathrm{p}<0.001$ ) (fig 2). $\Delta \mathrm{Ts}$-SD exhibited a significant correlation with $\triangle \mathrm{LVEF}$ in the entire population $(\mathrm{r}=-0.43$, $p=0.005$ ) (fig 3). Changes in $S^{\prime}$ were comparable between the two groups $(-0.35(1.31) \mathrm{cm} / \mathrm{s}$ in the cell infusion group vs $-0.20(0.89) \mathrm{cm} / \mathrm{s}$ in the control group, $p=0.13)$, indicating no significant difference between the two groups in terms of the alteration in LV contractility.

Inter-observer and intra-observer variability of Ts-SD measurements showed excellent agreements (inter-observer correlation: $\mathrm{r}=0.97, \mathrm{SEE}=12.3, \mathrm{p}<0.001$ and intra-observer correlation: $r=0.99$, $\mathrm{SEE}=8.1, \mathrm{p}<0.001)$.

\section{Determinants of LV systolic function and LV synchronicity improvements}

To elucidate independent determinants of LVEF improvement, we performed multivariate analysis and included age, gender, the group factor (control vs cell infusion), medications used, hypertension, diabetes, changes of infarct volume, baseline LVEF, baseline LV end-systolic volume, baseline Ts-SD, baseline ORS duration, $\Delta S^{\prime}$ and $\Delta \mathrm{Ts}-\mathrm{SD}$ as independent variables (no change ( $\triangle \mathrm{LVEF}<5 \%$ ) or an aggravation of LVEF were coded as 0 and an LVEF improvement ( $\triangle \mathrm{LVEF} \geqslant 5 \%$ ) as 1$) . \Delta \mathrm{Ts}-\mathrm{SD}$ and baseline LVEF were emerging variables identified as independent determinants for LVEF improvement (table 3).

To identify determinants of LV dyssynchrony improvement, we again performed multivariate analysis. The dependent variable was $\Delta \mathrm{Ts}$-SD (no change or aggravation of $\mathrm{Ts}-\mathrm{SD}$ $(\Delta \mathrm{Ts}-\mathrm{SD} \geqslant 0)$ was coded as 0 and Ts-SD improvement $(\Delta \mathrm{Ts}-\mathrm{SD}$ $<0)$ as 1). Independent variables were age, gender, the group factor, medications used, hypertension, diabetes, changes of infarct volume, baseline LVEF, baseline LV end-systolic volume, baseline Ts-SD, baseline ORS duration, $\Delta S^{\prime}$ and $\Delta L V E F$. The determinants of $\Delta \mathrm{Ts}$-SD improvement were baseline Ts-SD and 
Table 1 Baseline clinical, echocardiographic, and cardiac magnetic resonance imaging (MRI) data according to either the presence or absence of cell infusion therapy or the response of Ts-SD after 6-month follow-up

\begin{tabular}{|c|c|c|c|c|c|c|}
\hline Subjects & Control & Cell infusion & p Value & Responders* & Non-responders* & p Value \\
\hline Number & 20 & 20 & & 24 & 16 & \\
\hline Age (years) & $57.1(11.9)$ & $56.6(13.1)$ & 0.90 & $56.1(13.0)$ & $57.9(11.7)$ & 0.66 \\
\hline Male, n (\%) & $16(80)$ & 17 (85) & 1.0 & $20(83)$ & $13(81)$ & 1.0 \\
\hline Diabetes, n (\%) & $7(35)$ & $5(25)$ & 0.49 & $7(30)$ & $5(31)$ & 0.89 \\
\hline ORS duration (ms) & $93.0(17.2)$ & $96.5(22.3)$ & 0.58 & $93.8(21.6)$ & $96.3(17.1)$ & 0.70 \\
\hline Primary reperfusion, n (\%) & $13(65)$ & $10(50)$ & 0.34 & $15(62)$ & $8(50)$ & 0.43 \\
\hline Infarct related artery (LAD:LCX:RCA) & $8: 2: 10$ & $13: 3: 4$ & 0.14 & $15: 2: 7$ & $6: 3: 7$ & 0.28 \\
\hline \multicolumn{7}{|l|}{ Cardiac MRI parameters } \\
\hline LVESV $(\mathrm{mll}) \dagger$ & $65.5(36.5)$ & $64.7(22.0)$ & 0.93 & $62.9(22.0)$ & $68.5(39.2)$ & 0.56 \\
\hline LV EDV $(\mathrm{ml}) \dagger$ & $134.6(32.6)$ & $134.7(32.4)$ & 0.99 & $131.5(30.7)$ & $139.3(34.5)$ & 0.46 \\
\hline LV EF $(\%) \dagger$ & $53.2(15.7)$ & $52.6(9.1)$ & 0.89 & $52.7(11.0)$ & $53.3(15.2)$ & 0.70 \\
\hline Infarct volume $(\mathrm{ml}) \dagger$ & $25.3(15.5)$ & $31.7(20.3)$ & 0.28 & $28.1(16.9)$ & $28.9(20.1)$ & 0.89 \\
\hline DT (ms) & $194.28(53.01)$ & $180.85(36.24)$ & 0.36 & $177.87(41.63)$ & $202.63(47.7)$ & 0.10 \\
\hline $\mathrm{S}^{\prime}(\mathrm{cm} / \mathrm{s})$ & $5.45(1.89)$ & $5.26(1.40)$ & 0.72 & $5.47(1.47)$ & $5.19(1.92)$ & 0.61 \\
\hline Ts-SD & $98.20(52.83)$ & $108.00(50.11)$ & 0.55 & $113.50(47.25)$ & $87.50(54.08)$ & 0.12 \\
\hline Ts-SD $>46$ ms, n (\%) & $15(75 \%)$ & $17(85 \%)$ & 0.70 & $21(88 \%)$ & $11(68.8 \%)$ & 0.23 \\
\hline Cell infusion, $\mathrm{n}(\%)$ & $0(0 \%)$ & $20(100 \%)$ & $<0.001$ & $17(71 \%)$ & $3(19 \%)$ & 0.003 \\
\hline \multicolumn{7}{|l|}{ Medications used, n (\%) } \\
\hline$\beta$-blockers & $14(70 \%)$ & $17(85 \%)$ & 0.45 & $18(75 \%)$ & $13(81 \%)$ & 0.72 \\
\hline ACEls or ARBs & $18(90 \%)$ & $19(95 \%)$ & 1.0 & $23(96 \%)$ & $14(88 \%)$ & 0.55 \\
\hline Aspirin or clopidogrel & $20(100 \%)$ & $20(100 \%)$ & 1.0 & $24(100 \%)$ & $16(100 \%)$ & 1.0 \\
\hline Statins & $18(90 \%)$ & $16(80 \%)$ & 0.66 & $20(83 \%)$ & $14(88 \%)$ & 1.0 \\
\hline
\end{tabular}

LV, left ventricular; ESV, end-systolic volume; EDV, end-diastolic volume; EF, ejection fraction; DT, deceleration time of E wave; S', mean peak systolic myocardial velocity; Ts-SD, standard deviation of the time to peak myocardial velocities; ACEls, angiotensin converting enzyme inhibitors; ARBs, angiotensin receptor blockers.

${ }^{*}$ Responders were defined as patients with (Ts-SD at 6 months - Ts-SD at baseline $\left.(\Delta T s-S D)\right)<0$ and non-responders as those with $\Delta \mathrm{Ts}$-SD $\geqslant 0$.

$\dagger$ Measured by cardiac magnetic resonance imaging.

the group factor-that is, the performance of cell therapy (table 3).

\section{Correlation between LV synchronicity and exercise capacity}

Maximal exercise capacity in the control group did not improve significantly at the 6-month follow-up, whereas exercise capacity showed a significant improvement in the cell infusion group (table 2 ).

We also found that exercise capacity at the 6-month followup was significantly correlated with Ts-SD $(r=-0.54$, $p=0.001)$ but not with LVEF $(p=0.46$, fig 4$)$. In the cell infusion group, Ts-SD was also found to be strongly correlated with maximum exercise capacity $(r=-0.61, p=0.007)$. But, LVEF failed to show a significant correlation with maximum exercise capacity $(p=0.59)$.

There were no deaths, new MI, hospitalisation due to angina or heart failure or revascularisation during 6-month follow-up in either groups.

\section{DISCUSSION}

This study, for the first time, demonstrates that stem cell therapy improved LV dyssynchrony in systole after AMI, as

Table 2 Morphological and functional parameters at baseline and follow-up

\begin{tabular}{|c|c|c|c|c|c|c|}
\hline \multirow[b]{2}{*}{ Subjects } & \multirow{2}{*}{$\frac{\text { Control }}{\text { Baseline }}$} & \multirow{2}{*}{$\frac{\text { Control }}{\text { Follow-up }}$} & \multirow[b]{2}{*}{ p Value } & \multirow{2}{*}{$\begin{array}{l}\text { Cell infusion } \\
\text { Baseline }\end{array}$} & \multirow{2}{*}{$\frac{\text { Cell infusion }}{\text { Follow-up }}$} & \multirow[b]{2}{*}{ p Value } \\
\hline & & & & & & \\
\hline LV EF (\%)* & $53.2(15.7)$ & $53.4(12.6)$ & 0.88 & $52.6(9.1)$ & $59.4(10.6)$ & 0.004 \\
\hline LV ESV $(\mathrm{ml})^{*}$ & $65.5(36.5)$ & $69.8(37.3)$ & 0.20 & $64.7(22.0)$ & $61.6(31.7)$ & 0.56 \\
\hline LV EDV $(\mathrm{ml})^{*}$ & $134.6(32.6)$ & $142.4(38.5)$ & 0.12 & $134.7(32.4)$ & $145.0(38.0)$ & 0.12 \\
\hline LV stroke volume $(\mathrm{ml})^{*}$ & $69.0(13.6)$ & $72.5(12.2)$ & 0.24 & $70.0(17.6)$ & $83.3(15.6)$ & 0.005 \\
\hline Infarct volume $(\mathrm{ml})^{*}$ & $25.3(15.5)$ & $21.9(9.9)$ & 0.079 & $31.4(20.8)$ & $23.6(16.5)$ & 0.008 \\
\hline Ts-SD & $98.2(52.8)$ & $103.2(50.1)$ & 0.59 & $108.0(50.1)$ & $63.0(54.9)$ & $<0.001$ \\
\hline $\mathrm{S}^{\prime}(\mathrm{cm} / \mathrm{s})$ & $5.5(1.9)$ & $5.1(1.4)$ & 0.25 & $5.26(1.40)$ & $5.5(1.2)$ & 0.70 \\
\hline QRS duration (ms) & $93.0(17.2)$ & $93.0(13.4)$ & 1.0 & $96.5(22.3)$ & $98.5(20.3)$ & 0.32 \\
\hline Maximal exercise capacity (METs) & $9.3(2.8)$ & $9.8(3.5)$ & 0.35 & $8.8(2.8)$ & $10.1(2.4)$ & 0.002 \\
\hline
\end{tabular}

* Measured by cardiac magnetic resonance imaging.

LV, left ventricular; ESV, end-systolic volume; EDV, end-diastolic volume; EF, ejection fraction; Ts-SD, standard deviation of the time to peak myocardial velocities; $\mathrm{S}^{\prime}$, mean peak systolic myocardial velocity. 
Table 3 Determinants for the improvement of the left ventricular ejection fraction or synchronicity

\begin{tabular}{|c|c|c|c|c|c|c|c|c|c|}
\hline \multicolumn{5}{|c|}{ Determinants for Ts-SD improvement } & \multicolumn{5}{|c|}{ Determinants for LVEF improvement } \\
\hline Independent factors & $\begin{array}{l}\text { Exponential } \\
\text { coefficient }(\beta)\end{array}$ & $\begin{array}{l}\text { Wald } \\
\text { statistics }\end{array}$ & p Value & $95 \% \mathrm{Cl}$ & Independent factors & $\begin{array}{l}\text { Exponential } \\
\text { coefficient }(\beta)\end{array}$ & $\begin{array}{l}\text { Wald } \\
\text { statistics }\end{array}$ & p Value & $95 \% \mathrm{Cl}$ \\
\hline Age & 0.961 & 1.425 & 0.233 & 0.899 to 1.026 & Age & 1.025 & 1.512 & 0.219 & 0.985 to 1.067 \\
\hline $\mathrm{DM}$ & 2.693 & 0.877 & 0.349 & 0.339 to 21.408 & $\mathrm{DM}$ & 1.365 & 0.103 & 0.748 & 0.205 to 9.091 \\
\hline HT & 0.666 & 0.146 & 0.702 & 0.0830 to 5.342 & HT & 1.263 & 0.055 & 0.814 & 0.181 to 8.832 \\
\hline ACEI or ARB & 7.128 & 3.221 & 0.073 & 0.834 to 60.888 & ACEI or ARB & 0.689 & 0.057 & 0.811 & 0.032 to 14.745 \\
\hline QRS duration & 1.023 & 0.820 & 0.365 & 0.970 to 1.088 & QRS duration & 0.991 & 0.136 & 0.712 & 0.945 to 1.039 \\
\hline LV EF (baseline) & 1.012 & 0.041 & 0.840 & 0.903 to 1.134 & LV EF (baseline) & 0.977 & 5.912 & 0.015 & 0.959 to 0.996 \\
\hline LV ESV (baseline) & 0.978 & 1.273 & 0.259 & 0.940 to 1.017 & LV ESV (baseline) & 0.990 & 0.795 & 0.373 & 0.968 to 1.012 \\
\hline Ts-SD (baseline) & 1.019 & 4.595 & 0.032 & 1.002 to 1.037 & Ts-SD (baseline) & 0.998 & 0.039 & 0.843 & 0.975 to 1.021 \\
\hline$\Delta$ infarct volume & 1.041 & 0.536 & 0.464 & 0.935 to 1.159 & $\Delta$ infarct volume & 0.929 & 2.998 & 0.083 & 0.854 to 1.010 \\
\hline$\Delta \mathrm{LV} E F$ & 1.094 & 1.353 & 0.245 & 0.940 to 1.273 & $\Delta \mathrm{Ts}-\mathrm{SD}$ & 0.982 & 3.930 & 0.047 & 0.965 to 0.999 \\
\hline
\end{tabular}

DM, diabetes mellitus; HT, hypertension; ACEls, angiotensin converting enzyme inhibitors; ARBs, angiotensin receptor blockers; LV, left ventricular; EF, ejection fraction; ESV, endsystolic volume; $\mathrm{S}^{\prime}$, mean peak systolic myocardial velocity; Ts-SD, standard deviation of the time to peak myocardial velocities.

evidenced by a significant reduction in Ts-SD (fig 5). Moreover, Ts-SD was found to be correlated with exercise capacity 6 months after stem cell therapy, suggesting that a restoration of LV dyssynchrony is related to an improved exercise capacity.

\section{LV dyssynchrony and its assessment with tissue synchronisation imaging}

The presence of LV dyssynchrony poses a higher risk of functional worsening and an important predictor of poor outcome independent of LVEF and other clinical factors. ${ }^{11}{ }^{12}$ Thus, the correction of LV dyssynchrony may be translated to a favourable prognosis ${ }^{13}{ }^{14}$ and the identification of LV mechanical dyssynchrony is of crucial importance.

Echocardiography has been the cornerstone for serial assessment of systolic dyssynchrony. Most of all, DTI has been the most widely used method with its high temporal resolution, high reproducibility and its ability to define regional timing and contractility. TSI is a novel echocardiographic imaging tool derived from DTI velocity data images. Previous studies have demonstrated the usefulness of TSI technique in assessing LV dyssynchrony. ${ }^{810}{ }^{15}$ Among the parameters frequently used to assess LV dyssynchrony, Ts-SD

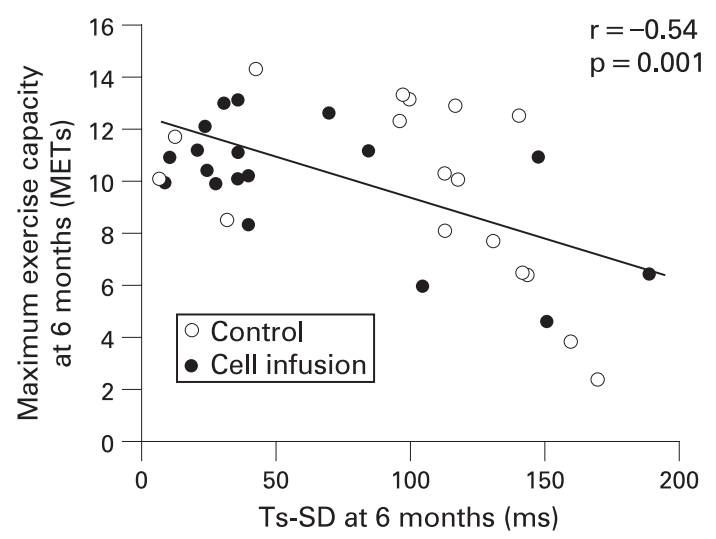

Figure 4 Correlation between the degree of LV dyssynchrony and exercise capacity at 6-month follow-up. Ts-SD measured 6 months after cell infusion showed a good correlation with maximum exercise capacity in all study subjects $(r=-0.54, p=0.001)$. is an reliable and powerful index that embraces the information of all the $12 \mathrm{LV}$ segments, and thus Ts-SD seems to be a more useful surrogate for LV dyssynchrony in patients with ischaemic cardiomyopathy. This concept was evidently supported by the earlier study by Yu et al, where Ts-SD is the single reliable index for LV dyssynchrony in ischaemic cardiomyopathy. ${ }^{10}$

\section{Restoration of LV synchronous contraction-a plausible mechanism of LVEF improvement with stem cell therapy}

Stem cell therapy was reported to be effective in numerous clinical trials with diverse stem cell collection and delivery protocol. ${ }^{6}{ }^{16-18}$ Schachinger et al reported improved LV systolic function in their randomised double-blind trial using intracoronary infusion of bone marrow-derived progenitor cells ${ }^{4}$ and Ince et al reported favourable results using peripheral stem cells by a mobilisation method with G-CSF. ${ }^{7}$ Most of them reported the improvement of LV systolic performance as shown by LVEF improvement and the mechanism of this phenomenon has been under discussion.

Although angiogenesis and myocardial regeneration have been suggested to account for improved cardiac systolic function by stem cell therapy, ${ }^{19}$ arguments were raised because only small numbers of stem cells could be observed in the longterm follow-up animal studies. Thus, direct myogenesis and angiogenesis were regarded as insufficient in explaining the functional improvements associated with stem cell therapy ${ }^{20}$ In these respects, the paracrine effects of transplanted stem cells were newly proposed as a reason for the improvements observed in clinical and preclinical studies, ${ }^{21}$ though this is mainly supported by circumstantial evidence. Additionally we do not have adequate methodology to evaluate myocardial regeneration and angiogenesis in a clinical setting. Only surrogate markers such as changes in infarct volume, myocardial perfusion score and global peak systolic myocardial velocity were used to evaluate the underlying mechanism of stem cell therapy. In fact, the underlying mechanism of this has not been clearly elucidated till now.

In the present study, we proposed changes of LV synchrony as a novel clinical parameter to explain the mechanism of stem cell therapy. We clearly demonstrated improvement of LV 
A

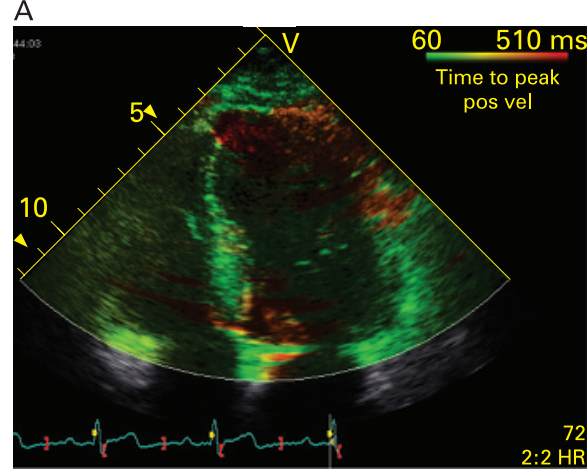

B

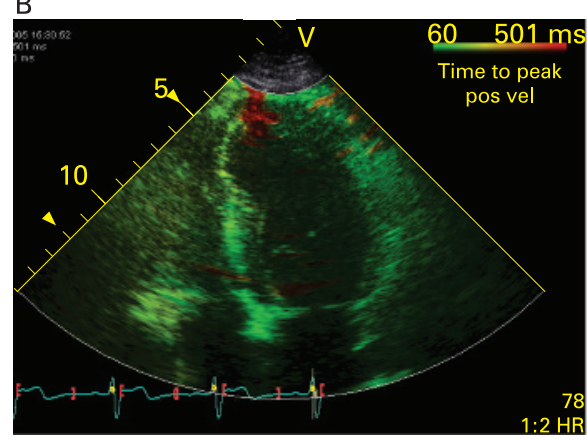

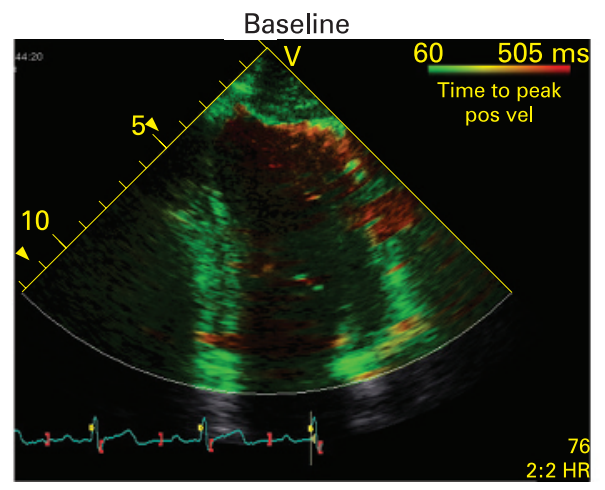

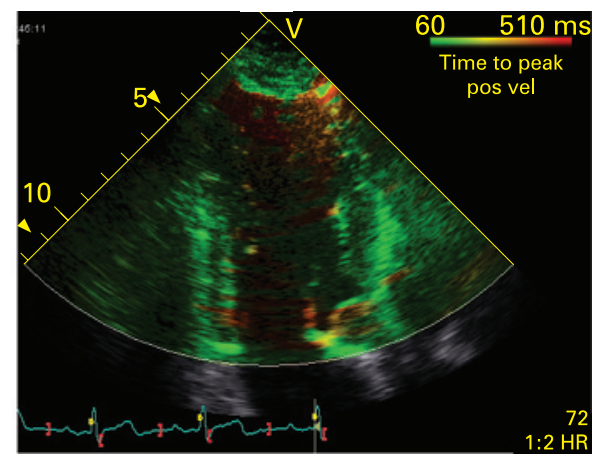

6 months after cell therapy

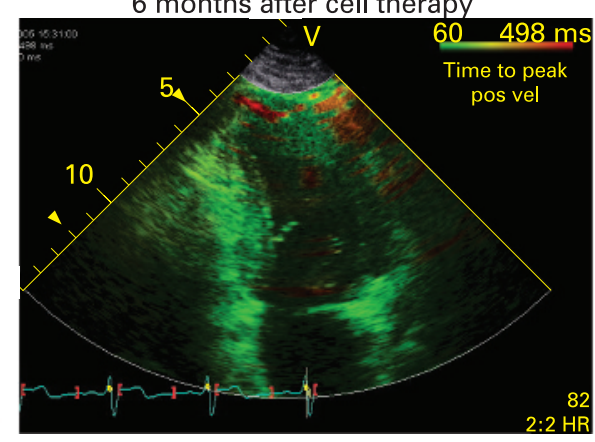

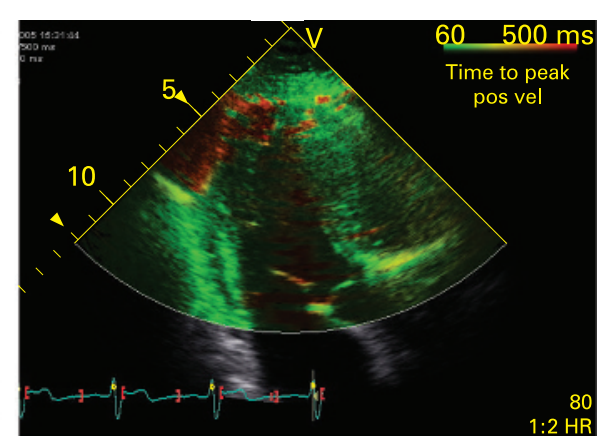

Figure 5 A representative example of tissue synchronisation imaging in the apical four-chamber, two-chamber and three-chamber views in a patient with AMI, who was assigned to the cell infusion group. Upper panel (A) represents images obtained at baseline. Red colours, indicating myocardial segments contracting in a dyssynchronous fashion, were superimposed on mid-anterolateral, anterior and apical walls. After 6 months of follow-up, the extent of the red coloured areas was remarkably diminished, as shown in lower panel (B), indicative of a significant improvement in left ventricular synchronicity with stem cell therapy.

synchrony after stem cell therapy as well as the close association between this improvement and LVEF enhancement. Of note, on multivariate analysis, LVEF improvement by stem cell therapy is independently related to a re-coordination of LV contraction as well as baseline LVEF rather than with to augmented contractility of the LV myocardium evaluated by S'. Although reductions in infarct volume were more prominent in the cell infusion group, infarct size did not play a vital part in altering the LVEF determinant or LV dyssynchrony, as demonstrated by multivariate analysis.

In hearts with asynchronous contraction, myocardial segments contracting earlier might add an additional burden to late-contracting myocardial segments beyond the preload naturally provided and, thus, cause substantial energy wastage during their contraction to overcome the increased preload, which could influence the contractility of late-contracting myocardium in view of the classic Frank-Starling mechanism locally operated. ${ }^{22}$ Moreover, asynchronous LV activation was shown to be associated with changes in regional myocardial blood flow and regional metabolism. ${ }^{23}$ Based on these previous observations, we can easily understand that asynchronous LV contraction could have a deleterious impact on the progression of underlying LV dysfunction, irrespective of its aetiology and consequently patients' prognosis. The alleviation of abnormally increased myocardial preload may have favourable repercussions on the recovery of regional myocardial function, which would in turn lead to LV reverse remodelling ${ }^{23}$ and LV systolic functional improvement. Accordingly, stem cell therapy, through LV resynchronisation, is likely to be a clinically relevant adjunctive therapy that enhances outcomes of patients with MI and LV dyssynchrony beyond that acquired by revascularisation.

\section{Restoration of LV dyssynchrony and exercise capacity}

Restoration of LV synchronicity with biventricular pacing improved symptoms and the functional capacities of patients with heart failure. ${ }^{24}$ In this study, the exercise capacity of patients in the cell infusion group improved at the 6-month follow-up, and Ts-SD, a surrogate index of LV synchronicity, but not LVEF, displayed a good correlation with maximal exercise capacity 6 months after stem cell therapy. This phenomenon suggests that the restoration of LV synchrony obtained by stem cell therapy is largely responsible for functional improvements in patients treated by stem cell therapy.

\section{Study limitations}

We cannot provide prognostic data owing to the relatively short follow-up period. However, earlier reports have suggested that restoring LV mechanical synchronicity mitigates patients' morbidity and mortality. ${ }^{13} 25$ Long-term follow-up results to show that the restored LV dyssynchrony after stem cell therapy led to a better prognosis are expected to be available in the near future. In addition, efforts to identify baseline clinical or echocardiographic parameters predictive of LV synchrony improvements after stem cell therapy are probably worthwhile, although the present study failed in this respect.

\section{CONCLUSIONS}

Treatment with peripheral blood stem cells mobilised by G-CSF offers a unique opportunity to restore LV synchronous contraction, which may be one of the underlying mechanisms for the improvement in LVEF and exercise capacity by stem cell therapy in patients with AMI. The hypothesis that the 
restoration of LV synchronicity after stem cell therapy may improve patients' long-term survival need to be clarified in future studies.

Acknowledgements: This study was approved by the institutional review board of Seoul National University Hospital.

Funding: This study was supported by a grant from the National Research Laboratory for Cardiovascular Stem Cell (HSK) and Innovative Research Institute for Cell Therapy (HSK, HJK).

Competing interests: None.

\section{REFERENCES}

1. Yu CM, Chau E, Sanderson JE, et al. Tissue Doppler echocardiographic evidence of reverse remodeling and improved synchronicity by simultaneously delaying regional contraction after biventricular pacing therapy in heart failure. Circulation 2002;105:438-45.

2. Abraham WT, Fisher WG, Smith AL, et al. Cardiac resynchronization in chronic heart failure. N Engl J Med 2002;346:1845-53.

3. Sutton MG, Plappert T, Hilpisch KE, et al. Sustained reverse left ventricular structural remodeling with cardiac resynchronization at one year is a function of etiology: quantitative Doppler echocardiographic evidence from the Multicenter InSync Randomized Clinical Evaluation (MIRACLE). Circulation 2006;113:266-72.

4. Schachinger V, Erbs S, Elsasser A, et al. Intracoronary bone marrow-derived progenitor cells in acute myocardial infarction. N Engl J Med 2006;355:1210-21.

5. Kang HJ, Kim HS, Zhang SY, et al. Effects of intracoronary infusion of peripheral blood stem-cells mobilised with granulocyte-colony stimulating factor on left ventricular systolic function and restenosis after coronary stenting in myocardial infarction: the MAGIC cell randomised clinical trial. Lancet 2004;363:751-6.

6. Kang HJ, Lee HY, Na SH, et al. Differential effect of intracoronary infusion of mobilized peripheral blood stem cells by granulocyte colony-stimulating factor on left ventricular function and remodeling in patients with acute myocardial infarction versus old myocardial infarction: the MAGIC Cell-3-DES randomized, controlled trial. Circulation 2006;114:1145-51.

7. Ince $\mathbf{H}$, Petzsch $\mathbf{M}$, Kleine HD, et al. Preservation from left ventricular remodeling by front-integrated revascularization and stem cell liberation in evolving acute myocardial infarction by use of granulocyte-colony-stimulating factor (FIRSTLINE-AMI). Circulation 2005:112:3097-106.

8. Gorcsan J 3rd, Kanzaki H, Bazaz R, et al. Usefulness of echocardiographic tissue synchronization imaging to predict acute response to cardiac resynchronization therapy. Am J Cardiol 2004;93:1178-81.

9. Yu CM, Fung JW, Zhang 0 , et al. Tissue Doppler imaging is superior to strain rate imaging and postsystolic shortening on the prediction of reverse remodeling in both ischemic and nonischemic heart failure after cardiac resynchronization therapy. Circulation 2004;110:66-73.
10. Yu CM, Zhang Q, Fung JW, et al. A novel tool to assess systolic asynchrony and identify responders of cardiac resynchronization therapy by tissue synchronization imaging. J Am Coll Cardiol 2005; 45:677-84.

11. Bader H, Garrigue S, Lafitte S, et al. Intra-left ventricular electromechanical asynchrony. A new independent predictor of severe cardiac events in heart failure patients. J Am Coll Cardiol 2004;43:248-56.

12. Fauchier L, Marie 0, Casset-Senon D, et al. Interventricular and intraventricular dyssynchrony in idiopathic dilated cardiomyopathy: a prognostic study with fourier phase analysis of radionuclide angioscintigraphy. J Am Coll Cardiol 2002;40:2022-30

13. Yu CM, Bleeker GB, Fung JW, et al. Left ventricular reverse remodeling but not clinical improvement predicts long-term survival after cardiac resynchronization therapy. Circulation 2005;112:1580-6.

14. Bax JJ, Bleeker GB, Marwick TH, et al. Left ventricular dyssynchrony predicts response and prognosis after cardiac resynchronization therapy. J Am Coll Cardiol 2004:44:1834-40.

15. Yu CM, Zhang Q, Chan YS, et al. Tissue Doppler velocity is superior to displacement and strain mapping in predicting left ventricular reverse remodelling response after cardiac resynchronisation therapy. Heart 2006;92:1452-6.

16. Engelmann MG, Theiss HD, Hennig-Theiss C, et al. Autologous bone marrow stem cell mobilization induced by granulocyte colony-stimulating factor after subacute STsegment elevation myocardial infarction undergoing late revascularization: final results from the G-CSF-STEMI (Granulocyte Colony-Stimulating Factor ST-Segment Elevation Myocardial Infarction) trial. J Am Coll Cardiol 2006;48:1712-21.

17. Lunde K, Solheim $S$, Aakhus $S$, et al. Intracoronary injection of mononuclear bone marrow cells in acute myocardial infarction. N Engl J Med 2006;355:1199-209.

18. Steinwender C, Hofmann R, Kammler J, et al. Effects of peripheral blood stem cell mobilization with granulocyte-colony stimulating factor and their transcoronary transplantation after primary stent implantation for acute myocardial infarction. Am Heart J 2006;151:1296 e7-13.

19. Orlic D, Kajstura J, Chimenti S, et al. Bone marrow cells regenerate infarcted myocardium. Nature 2001;410:701-5.

20. Murry CE, Soonpaa MH, Reinecke H, et al. Haematopoietic stem cells do not transdifferentiate into cardiac myocytes in myocardial infarcts. Nature 2004;428:664-8.

21. Gnecchi M, He H, Liang OD, et al. Paracrine action accounts for marked protection of ischemic heart by Akt-modified mesenchymal stem cells. Nat Med 2005;11:3678.

22. Breithardt $\mathbf{O A}$, Stellbrink $C$, Herbots $L$, et al. Cardiac resynchronization therapy can reverse abnormal myocardial strain distribution in patients with heart failure and left bundle branch block. J Am Coll Cardiol 2003;42:486-94.

23. Prinzen FW, Augustijn CH, Arts T, et al. Redistribution of myocardial fiber strain and blood flow by asynchronous activation. Am J Physiol 1990;259:H300-8.

24. Cleland JG, Daubert JC, Erdmann E, et al. The effect of cardiac resynchronization on morbidity and mortality in heart failure. N Engl J Med 2005;352:1539-49.

25. Yu CM, Fung WH, Lin $\mathrm{H}$, et al. Predictors of left ventricular reverse remodeling after cardiac resynchronization therapy for heart failure secondary to idiopathic dilated or ischemic cardiomyopathy. Am J Cardiol 2003;91:684-8. 